\title{
Heartbeat: prevalence and treatment of severe aortic stenosis in older patients
}

Aortic stenosis (AS) is common in the elderly with an increasing number of patients as our population ages but precise estimates of prevalence have been limited by inadequate diagnostic data in most clinical databases. In this issue of Heart, Owens and colleagues ${ }^{1}$ performed a targeted review of medical records for 5795 participants over age 65 years in the population based Cardiovascular Health Study to determine the frequency of moderate to severe AS. Over 25 years, the cumulative frequency of significant AS was $3.7 \%$ with $85 \%$ of these patients being hospitalised for severe AS, although only $1 / 2$ underwent aortic valve replacement. The adjusted incident of significant AS was higher in men, but lower in Blacks, compared with the rest of the study cohort (figure 1).

In an editorial, Iung and Arangalage $^{2}$ point out that this estimate of the community burden of AS is higher than previously reported, which has important implications for healthcare costs, particularly given the evidence that valve replacement is underused for this condition. More importantly, although currently the only effective treatment is valve replacement for severe AS, 'the hope of identifying a therapeutic target within the complex pathophysiology of AS, and subsequently a pharmacological treatment, seems hopefully within reach. In this setting, quality epidemiological studies are essential to better capture the true burden of the disease and help identify risk subsets of the population who may benefit from echocardiographic screening and early pharmacological intervention that may suspend or slow down the natural history of AS in the future.'

The ability to replace the aortic valve by a transcatheter, rather than surgical, approach has transformed the treatment of severe AS in the elderly, allowing effective therapy in many patients who might not have been

Division of Cardiology, University of Washington, Seattle, Washington, USA

Correspondence to Professor Catherine M Otto, Division of Cardiology, University of Washington, Seattle, WA 98195, USA; cmotto@uw.edu
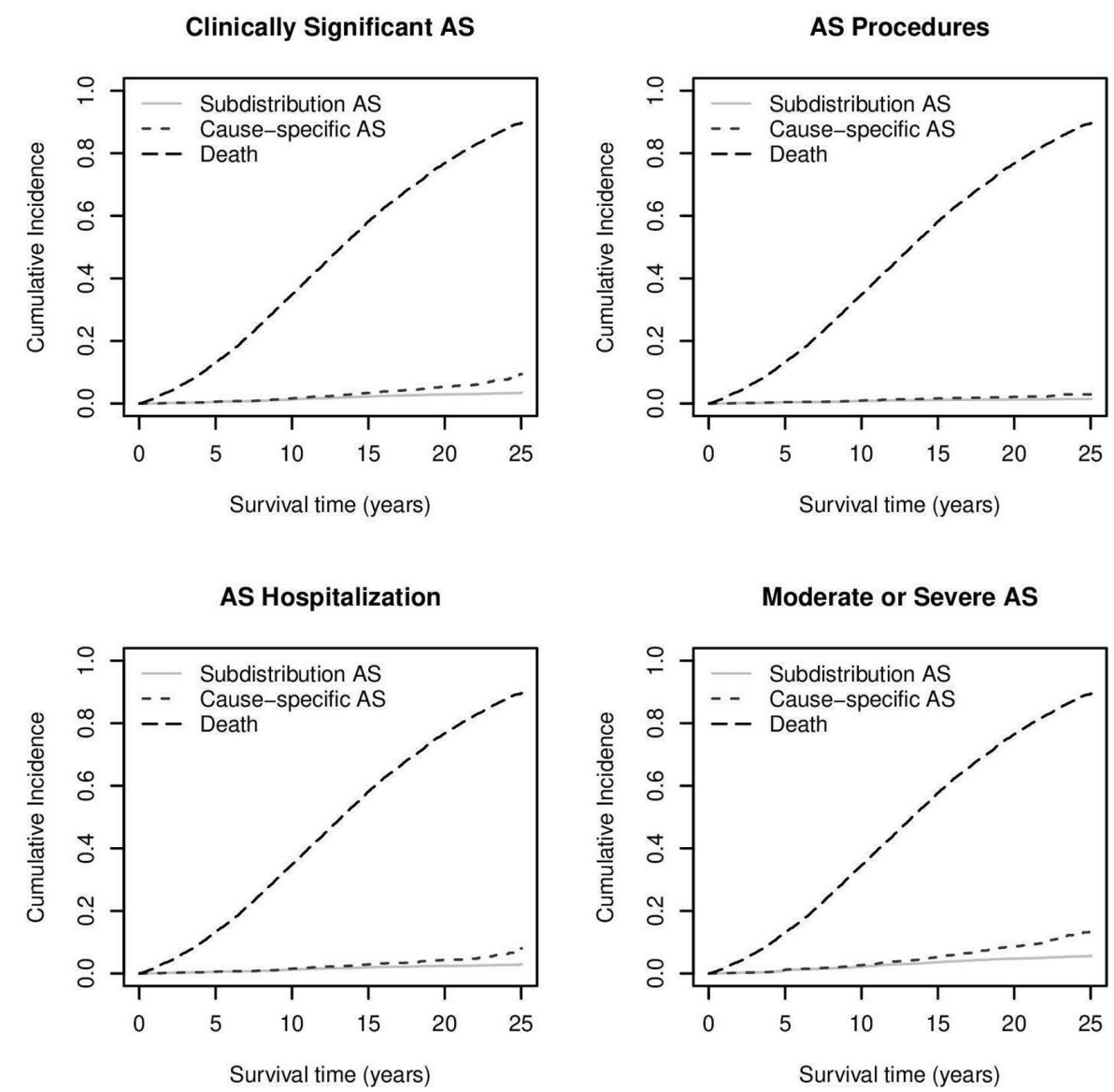

Figure 1 Cumulative incidence plots of AS events and death. Subdistribution and cause-specific AS refer to the plot for each aortic stenosis outcome calculated by subdistribution survival methods and cause specific survival methods, respectively. AS, aortic stenosis.

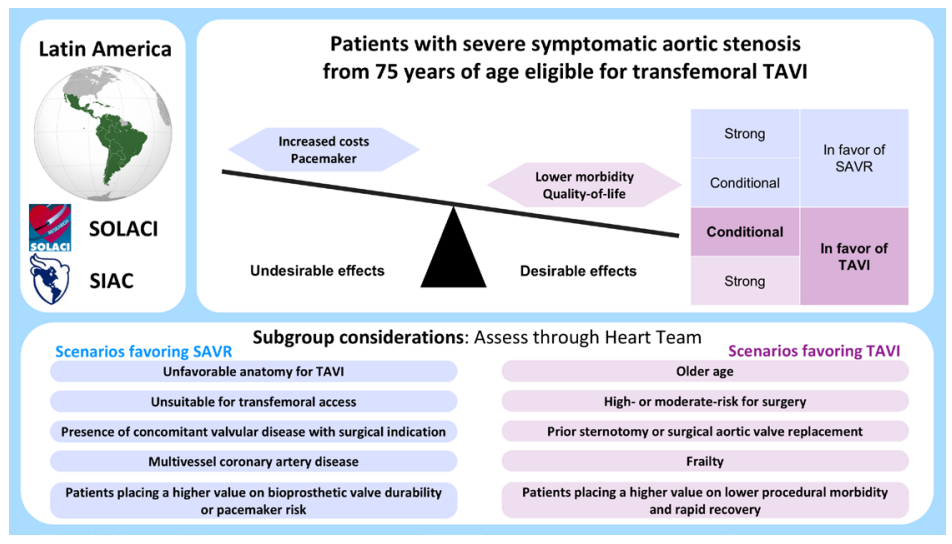

Figure 2 Latin American recommendations for subgroup considerations in in the decision for transcatheter aortic valve implantation (TAVI) versus surgical aortic valve replacement (SAVR). SIAC, Sociedad Interamericana de Cardiología; SOLACI, Sociedad Latino Americana de Cardiología Intervencionista. 

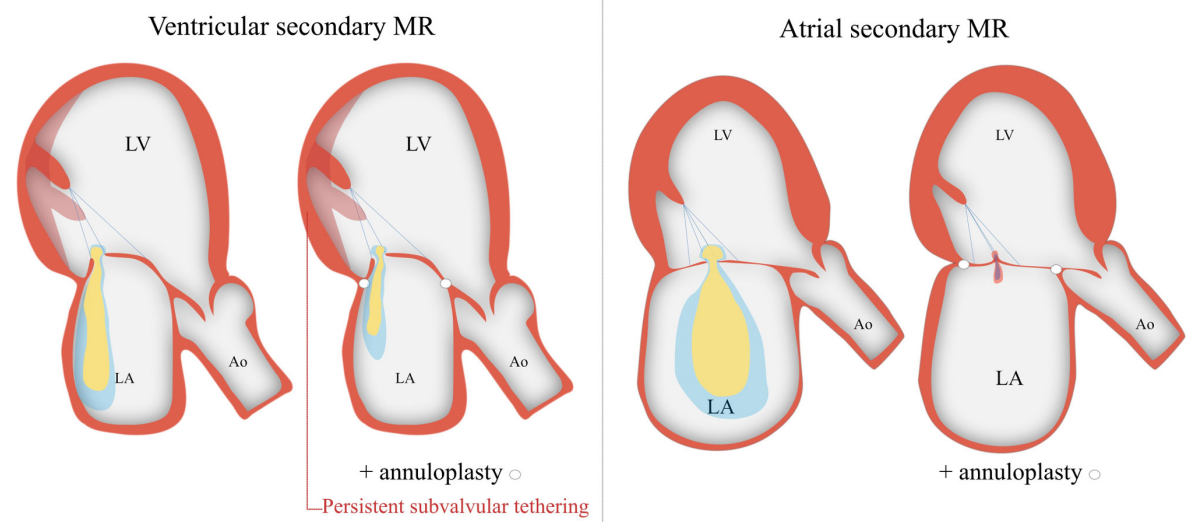

Figure 3 MVA to treat ventricular secondary MR versus atrial secondary MR schematic illustration showing persistent subannular leaflet tethering after annuloplasty to treat VSMR, opposed to improved leaflet coaptation in ASMR. ASMR, atrial secondary mitral regurgitation; LA, left atrium; LV, left ventricular; MR, mitral regurgitation; MVA, mitral valve annuloplasty; VSMR, ventricular secondary mitral regurgitation. treated in the past due to surgical risk, older age, comorbid conditions or frailty. However, this approach is costly so that guidelines developed by professional societies in high-income countries may not be applicable worldwide, requiring re-evaluation of recommendations for specific geographic regions. In this issue of Heart, Lamelas and colleagues ${ }^{3}$ present clinical practice guidelines for intervention for severe
AS in patients in Latin America. Their conditional recommendation, based on moderate certainty in the evidence, is that transcatheter valve implantation is preferred over surgical aortic valve replacement for patients with severe symptomatic AS living in Latin America who are 75 years of age or older. A detailed summary of the published evidence is provided in an online supplement along with a discussion of

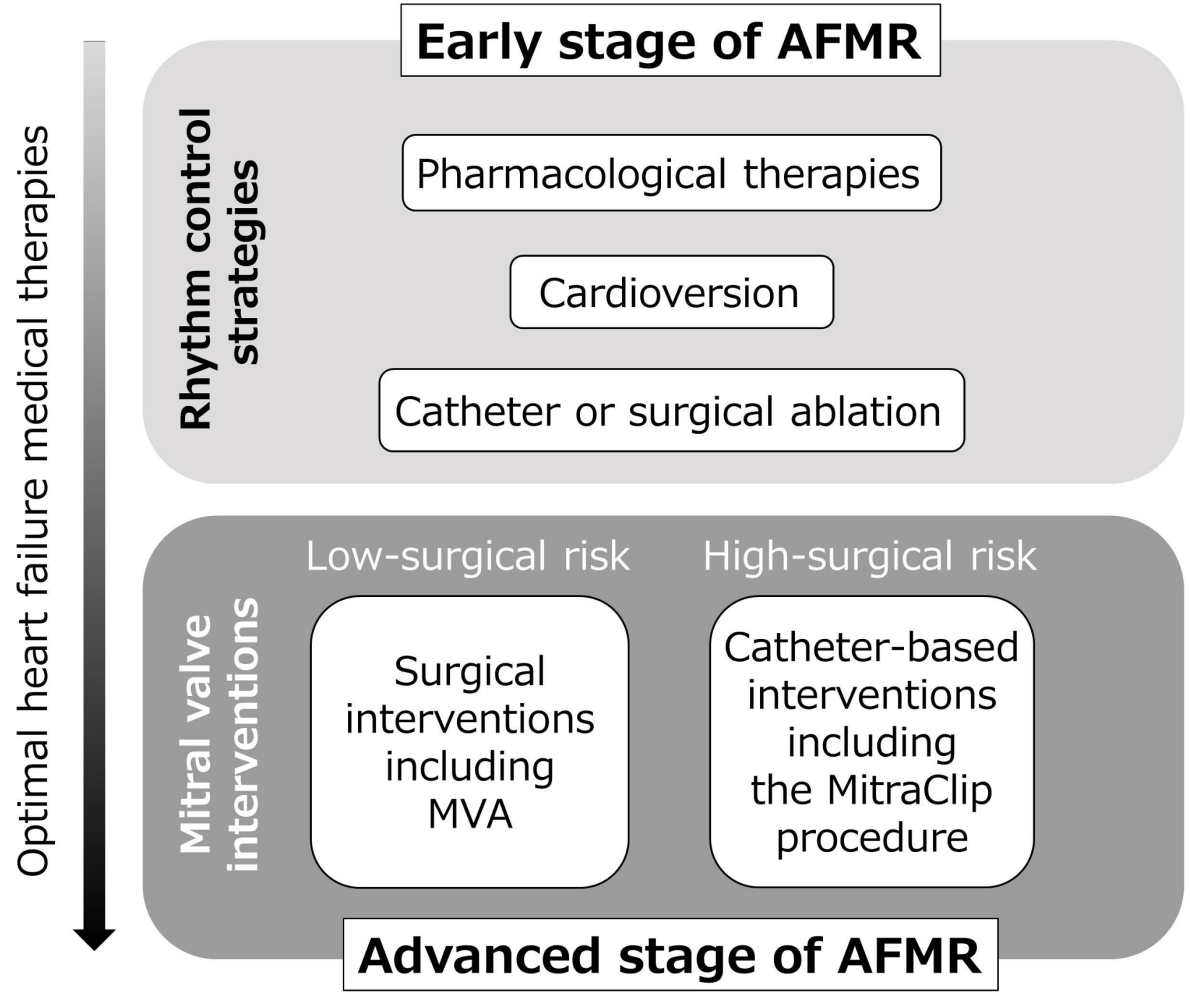

Figure 4 Currently available treatment options for management of AFMR. AFMR, atrial functional mitral regurgitation; MVA, mitral valve annuloplasty. subgroup consideration in this decision process (figure 2).

Newby and Mills ${ }^{4}$ 'commend and applaud the authors and the guideline development group for setting an example that many other guideline development groups would do well to follow.' 'The evidence tables give open and transparent assessments of the overall evidence and how they were evaluated and rated. They also give a guide as to the risks, benefits and potential biases as well as the importance of uncertainty and variability of the considered evidence. This is open, transparent and rigorous.' In addition, they support the concept that 'The inclusion of experts in the methods of systematic evidence evaluation as well as putting the patient at the centre of any recommendations is now mandatory.'

Management of secondary mitral regurgitation (MR) associated with excessive left atrial dilation, but normal left ventricular function, is challenging. Deferm and colleagues ${ }^{5}$ retrospectively analysed outcomes in patients with secondary MR who underwent surgical mitral valve annuloplasty. The 97 patients with atrial secondary MR, compared with 119 patients with ventricular secondary MR, were more often female ( $68 \%$ vs $34 \%$ ) with a higher prevalence of atrial fibrillation $(76 \%$ vs $34 \%$ ) but had a lower rate of recurrent significant MR at 2 years ( $7 \%$ vs $25 \%$ ) and a lower risk of death (adjusted HR $0.4395 \%$ CI 0.22 to $0.82, \mathrm{p}=0.011$ ). The authors propose the efficacy of annuloplasty for secondary MR reflects that differing pathophysiology of atrial versus ventricular dilation (figure 3 ).

In an editorial, Saito and colleagues ${ }^{6}$ discuss the pathophysiology of atrial functional (eg, secondary) MR (AFMR) which generally occurs in patients with heart failure with preserved ejection fraction and/or atrial fibrillation. In addition, the ambiguities surrounding this diagnosis are explored, as well as the association with prognosis and potential therapeutic options (figure 4). As they conclude: 'Further research is needed to determine a proper definition, elucidate its pathophysiology, understand the prognostic significance and establish appropriate treatment strategies for AFMR.'

The Education in Heart article in this issue $^{7}$ reviews management of calcified coronary artery lesions with a key point being the use of plaque modification of the calcified lesion before drug-eluting stent implantation. 


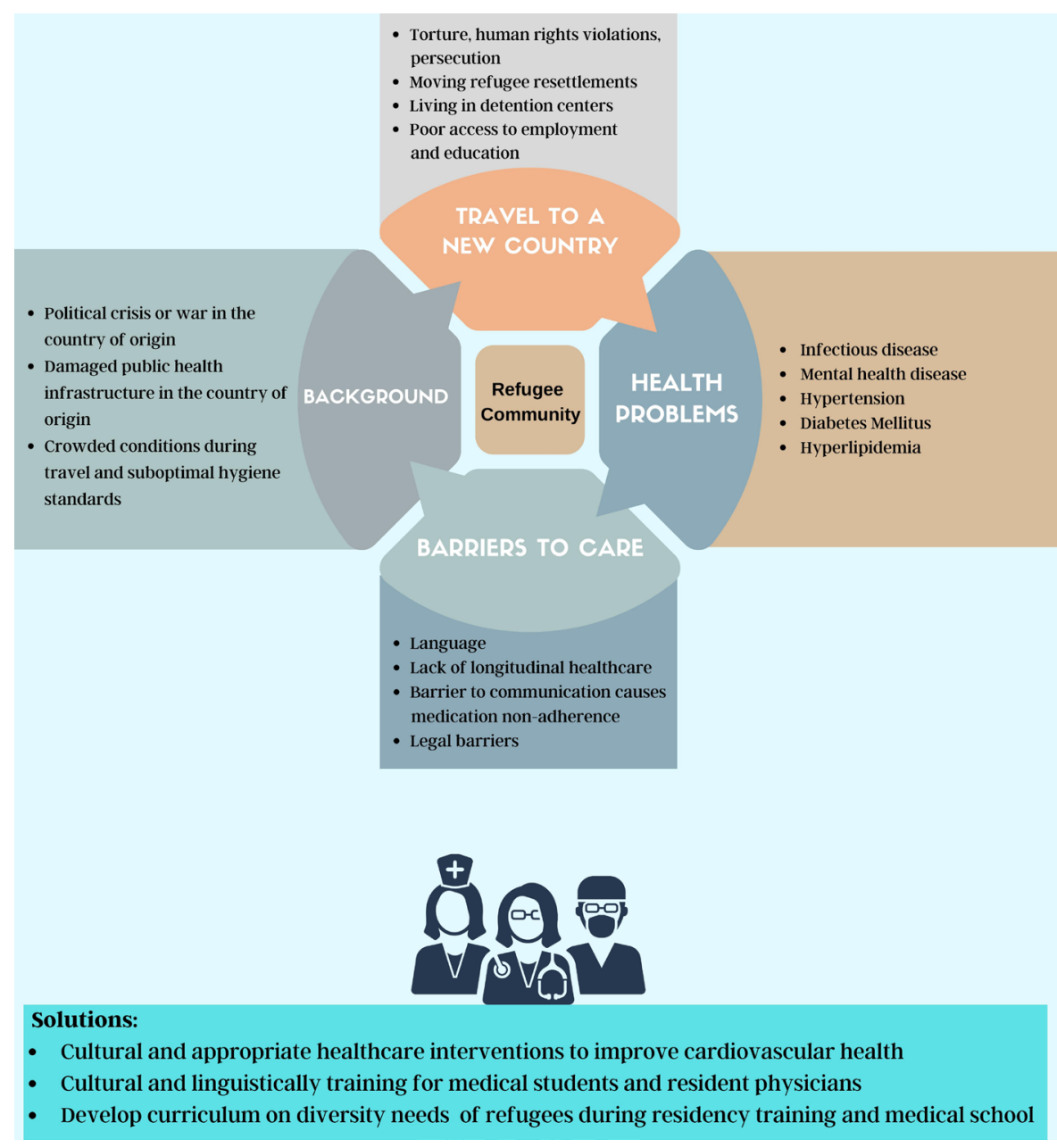

Figure 5 Risk factors for cardiovascular disease in refugee communities.

The Cardiology in Focus article in this issue $^{8}$ addresses the unique challenges in assessment and treatment of cardiovascular risk factors in refugee communities (figure 5).
Funding The authors have not declared a specific grant for this research from any funding agency in the public, commercial or not-for-profit sectors.

Competing interests None declared.
Patient and public involvement Patients and/ or the public were not involved in the design, or conduct, or reporting, or dissemination plans of this research.

Patient consent for publication Not required.

Provenance and peer review Commissioned; internally peer reviewed.

(C) Author(s) (or their employer(s)) 2021. No commercial re-use. See rights and permissions. Published by BMJ.

\section{(D) Check for updates}

To cite Otto CM. Heart 2021;107:1439-1441.

Heart 2021;107:1439-1441.

doi:10.1136/heartjnl-2021-320128

\section{ORCID iD}

Catherine M Otto http://orcid.org/0000-0002-05279392

\section{REFERENCES}

1 Owens DS, Bartz TM, Buzkova P, et al. Cumulative burden of clinically significant aortic stenosis in community-dwelling older adults. Heart 2021;107:1493-502.

2 lung B, Arangalage D. Community burden of aortic valve disease. Heart 2021;107:1446-7.

3 Lamelas P, Ragusa MA, Bagur R, et al. Clinical practice guideline for transcatheter versus surgical valve replacement in patients with severe aortic stenosis in Latin America. Heart 2021;107:1450-7.

4 Newby DE, Mills NL. Latin American guideline shows the way. Heart 2021;107:1442-3.

5 Deferm S, Bertrand PB, Verhaert D. Outcome and durability of mitral valve annuloplasty in atrial secondary mitral regurgitation. Heart 2021;107:1503-9.

6 Saito C, Minami Y, Hagiwara N. Treatment options for the management of atrial functional mitral regurgitation. Heart 2021;107:1448-9.

7 Barbato E. Contemporary management of calcified coronary lesions. Heart 2021;107:1510-7.

8 Santana J, Lemma A, O'Connor MH, et al. Understanding risk factors and preventive measures of cardiovascular disease in refugee communities. Heart 2021;107:1518-9. 